\title{
Amino Acid Based Ionic Liquids as Additives for the Separation of an Acetonitrile and Water Azeotropic Mixture: COSMO-RS Prediction and Experimental Verification
}

\author{
Tingting Li ${ }^{\dagger}$ Qing Yang,${ }^{\dagger}$ Hairong Ding,${ }^{\dagger}$ Jinlong Li, ${ }^{*}{ }^{\ddagger}$ Changjun Peng, ${ }^{* \dagger}$ and Honglai Liu ${ }^{\dagger}$ \\ $\dagger$ State Key Laboratory of Chemical Engineering and Department of Chemistry, and $\ddagger$ Key \\ Laboratory of Advanced Control and Optimization for Chemical Processes, Ministry of \\ Education, East China University of Science and Technology, Shanghai 200237, China
}

Procedures of interaction energy calculation and correlation of experimental data were provided. In Table S1, the detailed structures of all investigated anions were listed. In Tables S2 S4, van der Waals radii of the related atoms, stable energy of systems and interaction energy values were shown. In tables S5 S9, the isobaric VLE data of binary system for acetonitrile (1) + water (2), ternary systems for acetonitrile (1) + water (2) + [EMIM] [OAC] (3) and + [EMIM][Pro] (3) at fixed IL concentration 0.05 and 0.10 (mole fraction ), the parameters of Antoine constants and all correlation NRTL parameters are given, in which $T$ represents the equilibrium temperature corrected to $101.3 \mathrm{kPa} ; x_{1}$ represents the mole fraction of acetonitrile in liquid phase; $x_{3}$ represents the mole fraction of IL in liquid phase; $x_{1}{ }^{\prime}$ represents the mole fraction of acetonitrile in liquid phase without IL; $y_{1}$ represents the mole fraction of acetonitrile in vapor phase; $\alpha_{12}$ represents the relative volatility of acetonitrile. Optimized geometries, apparatus reliability test, VLE experimental data with NRTL results are shown in Figure S1-S4, respectively. This material is available free of charge via the internet at http://pubs.acs.org.

\section{Interaction Energy Calculation}

(1) Optimize molecular structures and calculate the stable energy of systems with Gaussian 03 based the DFT theory at the B3LYP/6-311g level.

(2) Calculate the interaction energy between ILs and the two solutes quantitatively by the expressions:

$$
\begin{aligned}
& I E_{\mathrm{CH}_{3} \mathrm{CN}-\mathrm{H}_{2} \mathrm{O}}=E_{\mathrm{CH}_{3} \mathrm{CN}+\mathrm{H}_{2} \mathrm{O}}-E_{\mathrm{CH}_{3} \mathrm{CN}}-E_{\mathrm{H}_{2} \mathrm{O}} \\
& I E_{\mathrm{CH}_{3} \mathrm{CN}-\mathrm{IL}}=E_{\mathrm{CH}_{3} \mathrm{CN}+\mathrm{IL}}-E_{\mathrm{CH}_{3} \mathrm{CN}}-E_{\mathrm{IL}} \\
& I E_{\mathrm{IL}-\mathrm{H}_{2} \mathrm{O}}=E_{\mathrm{IL}+\mathrm{H}_{2} \mathrm{O}}-E_{\mathrm{IL}}-E_{\mathrm{H}_{2} \mathrm{O}}
\end{aligned}
$$


where $I E$ is the interaction energy in $\mathrm{kJ} / \mathrm{mol}$, and $E$ is the stable energy of mixture or pure solution in $\mathrm{kJ} / \mathrm{mol}$.

\section{Correlation of Experimental Data}

(1) Neglect the non-ideality of vapor phase mixture and express the equilibrium relation by activity coefficient method as the following equation:

$$
P y_{i}=p_{i}^{S} x_{i} \gamma_{i}(i=1, . ., N)
$$

(2) Determine the parameters of A, B and C of antonine contants ${ }^{44}$

$$
\ln p^{S}=A-\frac{B}{T+C}
$$

Here, $P$ represents the total pressure; $P_{i}^{S}$ and $\gamma_{i}$ mean the saturation vapor pressure and the activity coefficient of component $i ; T$ is the corresponding temperature in $\mathrm{K}$.

(3) Establish an objective function based on NRTL model to determine the six energy parameters $\left(\Delta \mathrm{g}_{i j}, \Delta \mathrm{g}_{j i}\right)$ for each ternary mixture:

$$
F=\sqrt{\frac{5}{N} \sum_{i=1}^{N}\left(\frac{T^{c a l}-T^{\exp }}{T^{\exp }}\right)^{2}+\frac{1}{N} \sum_{i=1}^{N}\left(y^{\exp }-y^{c a l}\right)^{2}}
$$

where $N$ represents the number of experimental points; the superscripts 'cal' and 'exp' mean the NRTL correlated and experimental results.

(4) Enter experimental VLE data of acetonitrile and water in Matlab to fit the binary parameters of acetonitrile and water .

(5) Set the nonrandomness factors unknown in NRTL to 0.3 and enter experimental VLE data of acetonitrile (1) + water (2) + IL (3) in Matlab to fit the remaining model parameters. 
Table S1. List of anions

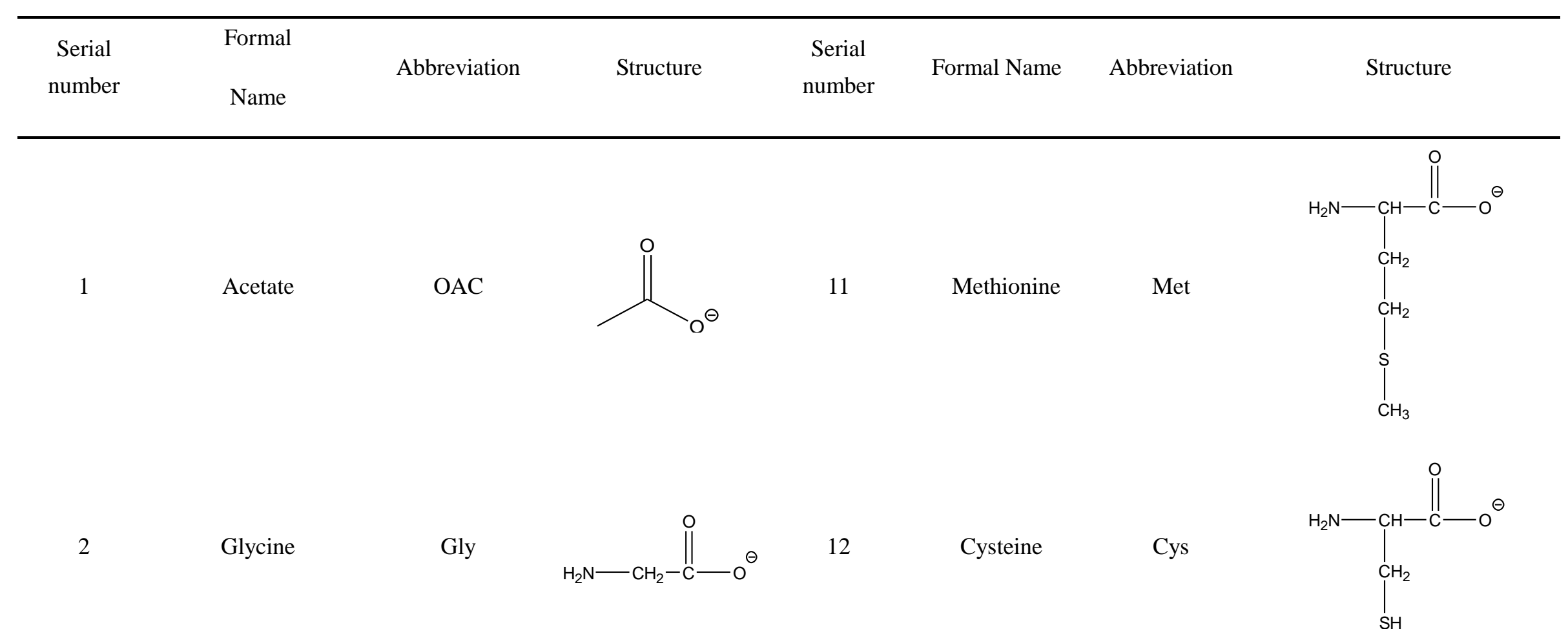


Pro

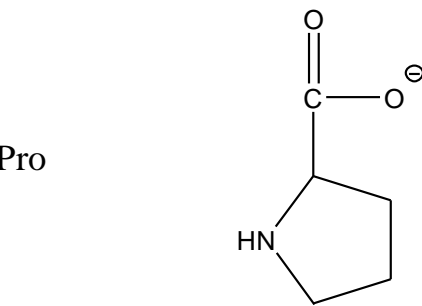

Histidine

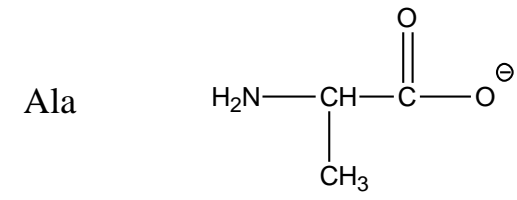

14
Glutamine

His

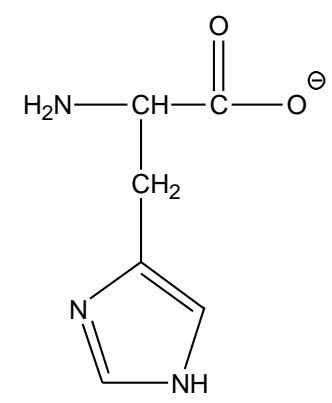

Gln

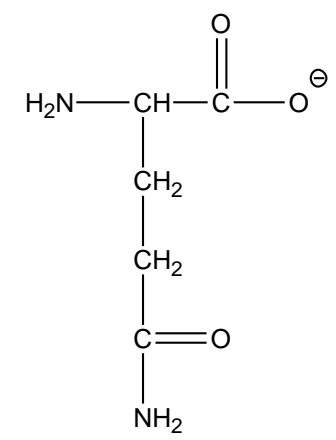


Lys

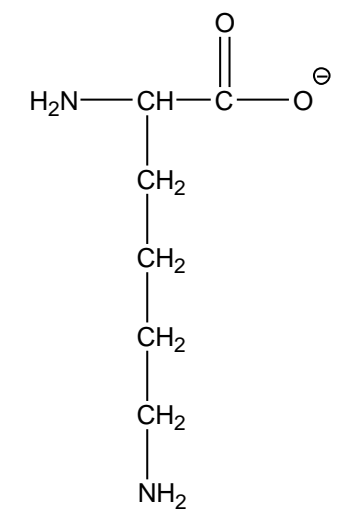

Thr

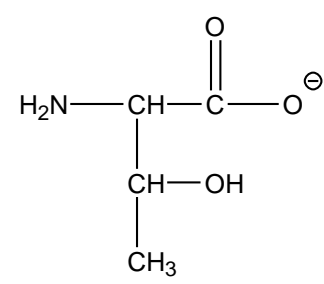

Valine

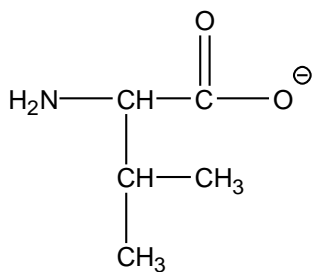

16

Serine

Ser

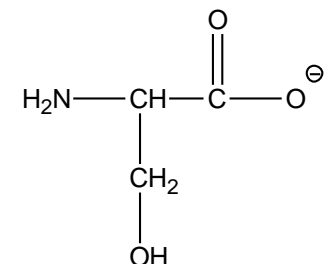

7

Isoleucine

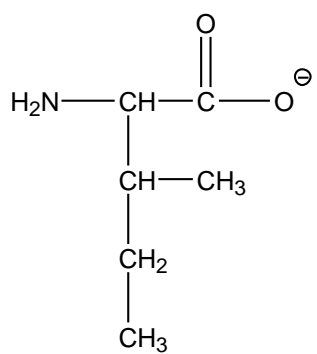

Glutamic acid

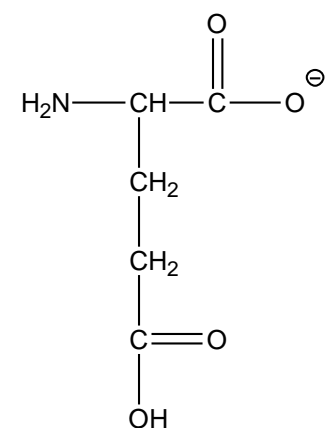



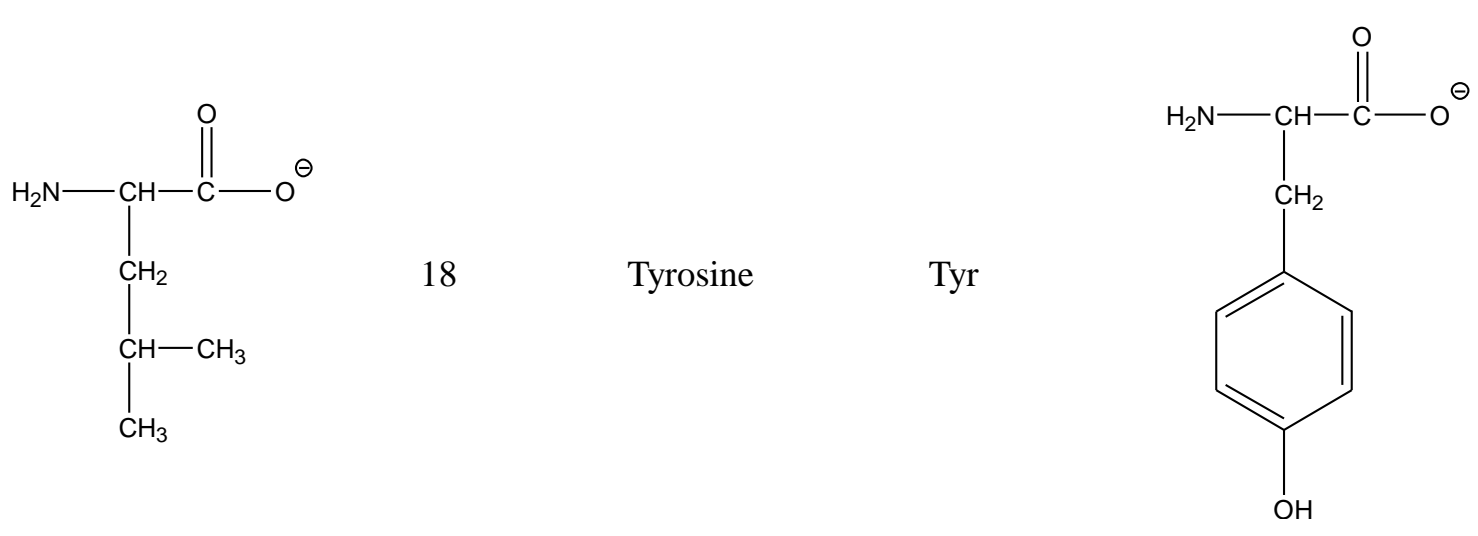

Aspartic acid
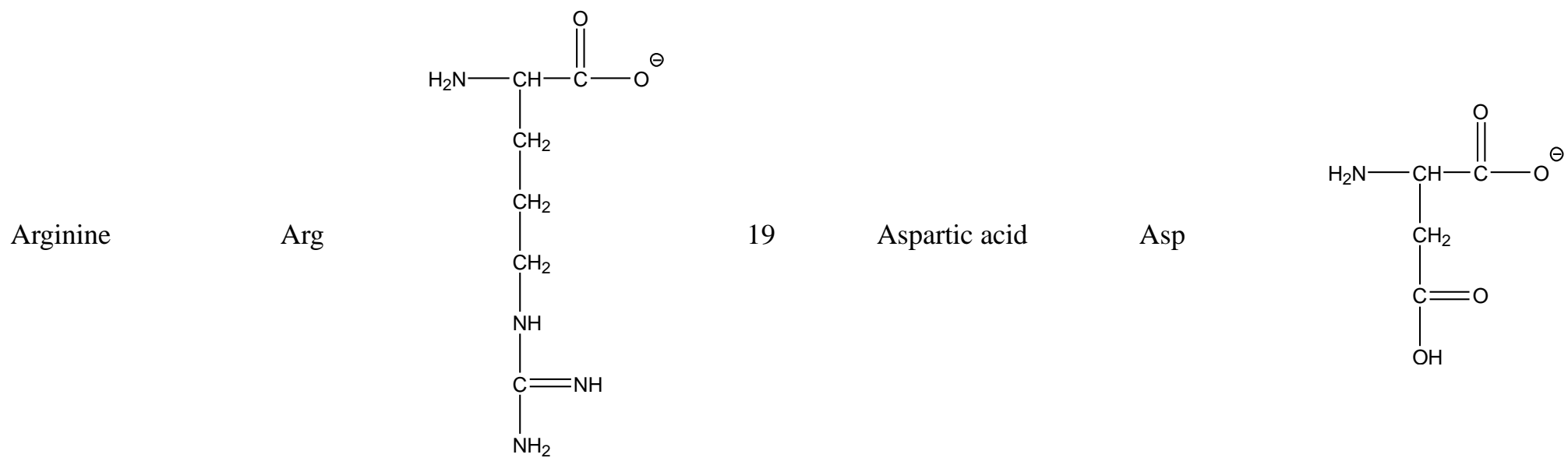
$10 \quad$ Phenylalanine

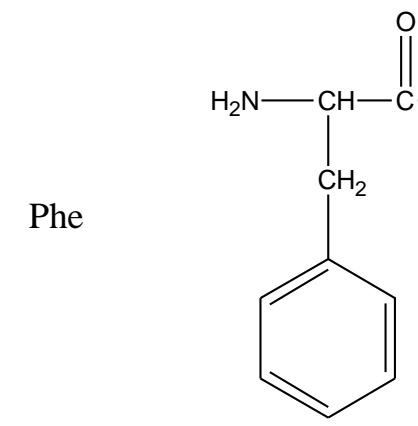


Table S2. van der Waals Radii of Atoms ${ }^{1}$

\begin{tabular}{ccccc}
\hline atom & $\mathrm{H}$ & $\mathrm{C}$ & $\mathrm{N}$ & $\mathrm{O}$ \\
\hline vdWradius/ $\AA$ & 1.20 & 1.70 & 1.55 & 1.52 \\
\hline
\end{tabular}

Table S3. System energy of acetonitrile(1)+water(2)+IL(3)

\begin{tabular}{|c|c|c|c|}
\hline system & energy/ $\mathrm{kJ} \cdot \mathrm{mol}^{-1}$ & system & energy $/ \mathrm{kJ} \cdot \mathrm{mol}^{-1}$ \\
\hline \multicolumn{4}{|c|}{ acetonitrile $(1)+$ water $(2)+[$ EMIM $][\mathrm{OAc}](3)$} \\
\hline $\mathrm{E}_{\mathrm{IL}}$ & -1503768.1820 & $\mathrm{E}_{\text {acetonitrile+IL }}$ & -1852060.9280 \\
\hline $\mathrm{E}_{\mathrm{water}}$ & -200513.6733 & $\mathrm{E}_{\mathrm{IL}+\text { water }}$ & -1704335.2210 \\
\hline $\mathrm{E}_{\text {acetonitrile }}$ & -348256.9791 & $\mathrm{E}_{\text {acetonitrile }}+_{\text {water }}$ & -548770.6435 \\
\hline \multicolumn{4}{|c|}{ acetonitrile $(1)+$ water $(2)+[$ EMIM $][$ Pro $](3)$} \\
\hline $\mathrm{E}_{\mathrm{IL}}$ & -1955174.3060 & $\mathrm{E}_{\text {acetonitrile+IL }}$ & -2303466.2630 \\
\hline $\mathrm{E}_{\text {water }}$ & -200513.6733 & $\mathrm{E}_{\mathrm{IL+water}}$ & -2155740.0680 \\
\hline $\mathrm{E}_{\text {acetonitrile }}$ & -348256.9791 & $\mathrm{E}_{\text {acetonitrile }}+_{\text {water }}$ & -548770.6435 \\
\hline
\end{tabular}

Table S4. Interaction energy of acetonitrile-IL and water-IL

\begin{tabular}{ccc}
\hline component & acetonitrile $/ \mathrm{kJ} \cdot \mathrm{mol}^{-1}$ & water $/ \mathrm{kJ} \cdot \mathrm{mol}^{-1}$ \\
\hline$[$ EMIM][OAC] & -35.7661 & -53.3656 \\
{$[$ EMIM][Pro] } & -34.9779 & -52.0887 \\
water & -23.2700 & - \\
\hline
\end{tabular}


Table S5 . Isobaric VLE data for acetonitrile (1) + water (2) at $101.3 \mathrm{kPa}^{\mathrm{a}}$

\begin{tabular}{ccccccccc}
\hline$T / \mathrm{K}$ & $x_{1}{ }^{\prime}$ & $y_{1}$ & $\alpha_{12}$ & $T / \mathrm{K}$ & $x_{1}$ & $y_{1}$ & $\alpha_{12}$ \\
\hline \multicolumn{3}{c}{ literature data ${ }^{2}$} & \multicolumn{5}{c}{ experiment data } \\
\hline 352.90 & 0.954 & 0.895 & 0.412 & 359.65 & 0.029 & 0.263 & 11.948 \\
351.36 & 0.906 & 0.826 & 0.492 & 354.25 & 0.093 & 0.505 & 9.950 \\
350.04 & 0.808 & 0.744 & 0.693 & 353.15 & 0.142 & 0.559 & 7.659 \\
349.77 & 0.717 & 0.705 & 0.944 & 351.75 & 0.254 & 0.617 & 4.731 \\
349.78 & 0.631 & 0.682 & 1.259 & 350.55 & 0.402 & 0.655 & 2.824 \\
349.91 & 0.556 & 0.669 & 1.611 & 349.75 & 0.527 & 0.673 & 1.847 \\
349.95 & 0.481 & 0.657 & 2.063 & 349.15 & 0.718 & 0.728 & 1.051 \\
350.01 & 0.412 & 0.646 & 2.601 & 349.95 & 0.856 & 0.761 & 0.536 \\
350.14 & 0.350 & 0.639 & 3.298 & 353.55 & 0.986 & 0.945 & 0.244 \\
349.60 & 0.293 & 0.636 & 4.226 & & & & \\
350.34 & 0.227 & 0.634 & 5.919 & & & & \\
350.87 & 0.184 & 0.621 & 7.250 & & & & \\
350.31 & 0.146 & 0.604 & 8.934 & & & & \\
352.08 & 0.073 & 0.543 & 13.923 & & & & \\
357.25 & 0.026 & 0.284 & 14.801 & & & & \\
\hline
\end{tabular}

${ }^{\mathrm{a}}$ Standard uncertainties $u$ are $u(T)=0.01 \mathrm{~K}, u(p)=0.01 \mathrm{kPa}$ and $u\left(x_{1}{ }^{\prime}\right)=u\left(x_{1}\right)=u\left(y_{1}\right)=u\left(\alpha_{12}\right)=0.0001$ 
Table S6 . Isobaric VLE data for acetonitrile (1) + water (2) + [EMIM][OAC] (3) at 101.3

\begin{tabular}{ccccccccc}
\multicolumn{8}{c}{$\mathbf{k P a}^{\mathbf{a}}$} \\
\hline$T / \mathrm{K}$ & $x_{1}{ }^{\prime}$ & $y_{1}$ & $\alpha_{12}$ & $T / \mathrm{K}$ & $x_{1}{ }^{\prime}$ & $y_{1}$ & $\alpha_{12}$ \\
356.40 & 0.080 & 0.528 & 12.822 & 360.18 & 0.053 & 0.397 & 11.731 \\
351.17 & 0.160 & 0.651 & 9.800 & 351.36 & 0.104 & 0.647 & 15.871 \\
349.76 & 0.245 & 0.694 & 6.987 & 350.28 & 0.133 & 0.704 & 15.510 \\
349.75 & 0.327 & 0.719 & 5.256 & 351.08 & 0.288 & 0.783 & 8.941 \\
349.83 & 0.412 & 0.744 & 4.161 & 351.27 & 0.375 & 0.818 & 7.484 \\
350.88 & 0.531 & 0.794 & 3.398 & 351.60 & 0.470 & 0.847 & 6.229 \\
352.38 & 0.601 & 0.806 & 2.757 & 352.04 & 0.512 & 0.855 & 5.644 \\
349.69 & 0.661 & 0.825 & 2.408 & 351.39 & 0.571 & 0.870 & 5.014 \\
351.77 & 0.778 & 0.867 & 1.859 & 351.97 & 0.632 & 0.892 & 4.793 \\
353.37 & 0.858 & 0.914 & 1.756 & 352.70 & 0.698 & 0.915 & 4.662 \\
354.01 & 0.909 & 0.944 & 1.710 & 353.28 & 0.802 & 0.947 & 4.396 \\
354.15 & 0.950 & 0.969 & 1.640 & 354.36 & 0.938 & 0.985 & 4.291 \\
\hline
\end{tabular}

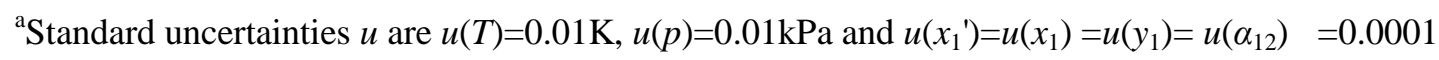


Table S7 . Isobaric VLE data for acetonitrile (1) + water (2) + [EMIM][Pro] (3) at $101.3 \mathrm{kPa}^{\mathrm{a}}$

\begin{tabular}{|c|c|c|c|c|c|c|c|}
\hline$T / \mathrm{K}$ & $x_{1}^{\prime}$ & $y_{1}$ & $\alpha_{12}$ & $T / \mathrm{K}$ & $x_{1}{ }^{\prime}$ & $y_{1}$ & $\alpha_{12}$ \\
\hline \multicolumn{4}{|c|}{$x_{3}=0.05$} & \multicolumn{4}{|c|}{$x_{3}=0.10$} \\
\hline 354.95 & 0.911 & 0.947 & 1.761 & 355.90 & 0.890 & 0.968 & 3.743 \\
\hline 352.61 & 0.805 & 0.875 & 1.700 & 355.68 & 0.804 & 0.943 & 4.047 \\
\hline 352.18 & 0.683 & 0.826 & 2.209 & 355.22 & 0.698 & 0.908 & 4.288 \\
\hline 350.13 & 0.605 & 0.792 & 2.482 & 354.96 & 0.573 & 0.873 & 5.102 \\
\hline 350.32 & 0.489 & 0.765 & 3.398 & 354.47 & 0.455 & 0.839 & 6.217 \\
\hline 350.18 & 0.452 & 0.749 & 3.624 & 355.75 & 0.388 & 0.815 & 6.939 \\
\hline 351.35 & 0.363 & 0.730 & 4.746 & 357.10 & 0.239 & 0.774 & 10.908 \\
\hline 350.71 & 0.298 & 0.716 & 5.929 & 365.40 & 0.127 & 0.705 & 16.472 \\
\hline 352.74 & 0.233 & 0.685 & 7.163 & 370.55 & 0.062 & 0.568 & 19.984 \\
\hline 354.87 & 0.177 & 0.639 & 8.208 & & & & \\
\hline 357.88 & 0.121 & 0.583 & 10.120 & & & & \\
\hline 363.10 & 0.077 & 0.473 & 10.796 & & & & \\
\hline
\end{tabular}

${ }^{\mathrm{a}}$ Standard uncertainties $u$ are $u(T)=0.01 \mathrm{~K}, u(p)=0.01 \mathrm{kPa}$ and $u\left(x_{1}{ }^{\prime}\right)=u\left(x_{1}\right)=u\left(y_{1}\right)=u\left(\alpha_{12}\right)=0.0001$ 
Table S8. Antoine constants ${ }^{3}$ of acetonitrile and water

\begin{tabular}{cccc}
\hline components & $A$ & $B$ & $C$ \\
acetonitrile & 14.27 & 2945.47 & -49.15 \\
water & 16.29 & 3816.44 & -46.13 \\
\hline
\end{tabular}


Table S9. Binary interaction parameters for NRTL Equation

\begin{tabular}{ccccc}
\hline component $i$ & component $j$ & $\left(\mathrm{~g}_{i j}-\mathrm{g}_{j j}\right) /\left(\mathrm{J}^{-\mathrm{mol}^{-1}}\right)$ & $\left(\mathrm{g}_{j i} \mathrm{~g}_{i i}\right) /\left(\mathrm{J} \cdot \mathrm{mol}^{-1}\right)$ & $\alpha_{i j}$ \\
\hline acetonitrile & water & 2215.3 & 5205.6 & 0.3545 \\
acetonitrile & {$[$ EMIM][OAC] } & -57244.3 & 4651.2 & 0.3000 \\
water $^{a}$ & {$[$ EMIM][OAC] } & -9881.7 & -8963.9 & 0.0930 \\
acetonitrile & {$[\mathrm{EMIM][Pro]}$} & -928.9 & -6026.6 & 0.3000 \\
water & {$[\mathrm{EMIM][Pro]}$} & -4487.3 & -12827.9 & 0.3000 \\
\hline
\end{tabular}

a. Parameters were calculated from ones published in literature ${ }^{4}$ 


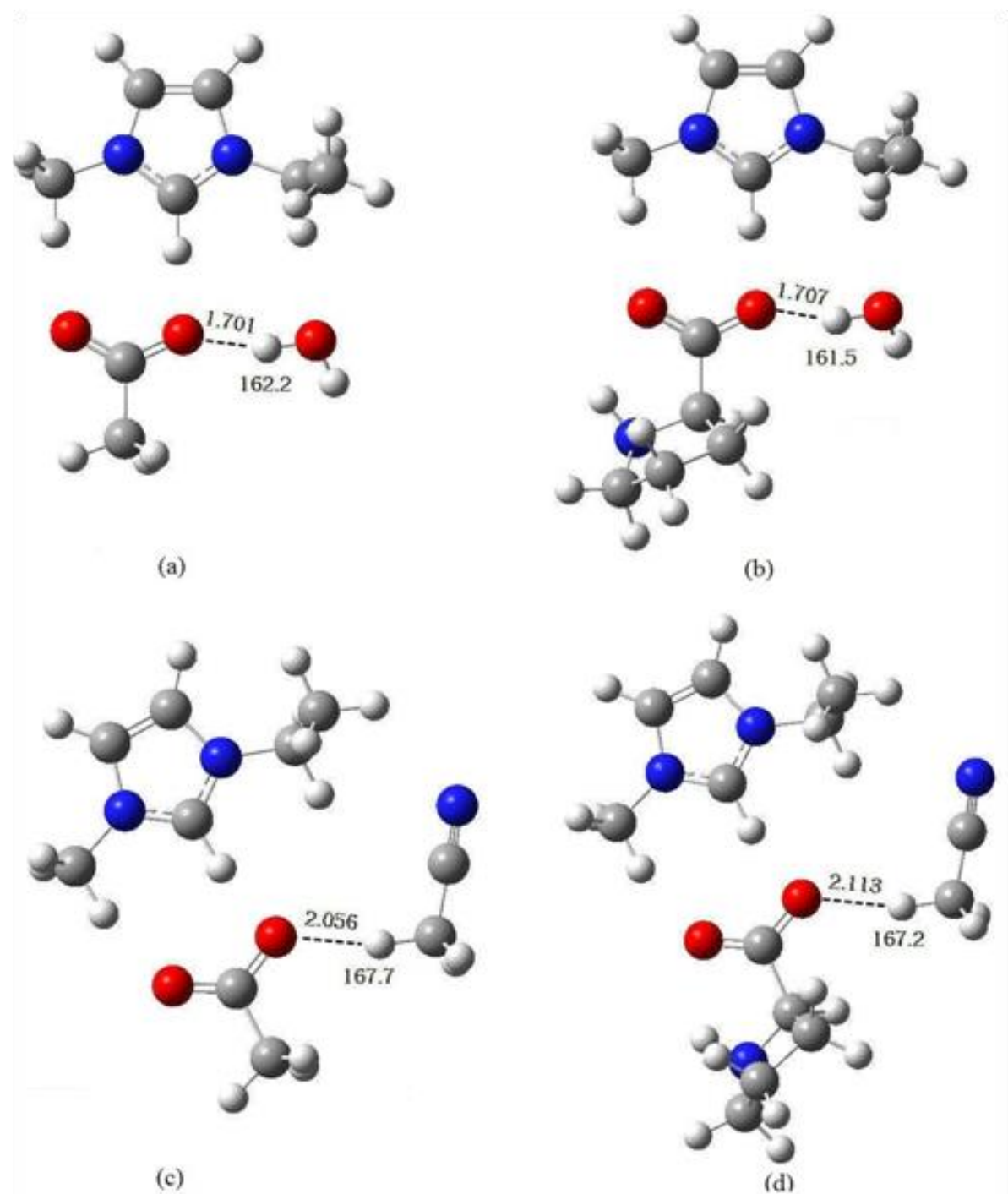

Figure S1. Optimized geometries: (a) water - [EMIM][OAC], (b) water- [EMIM][Pro], (c) acetonitrile - [EMIM][OAC] , and (d) acetonitrile - [EMIM][Pro]. 


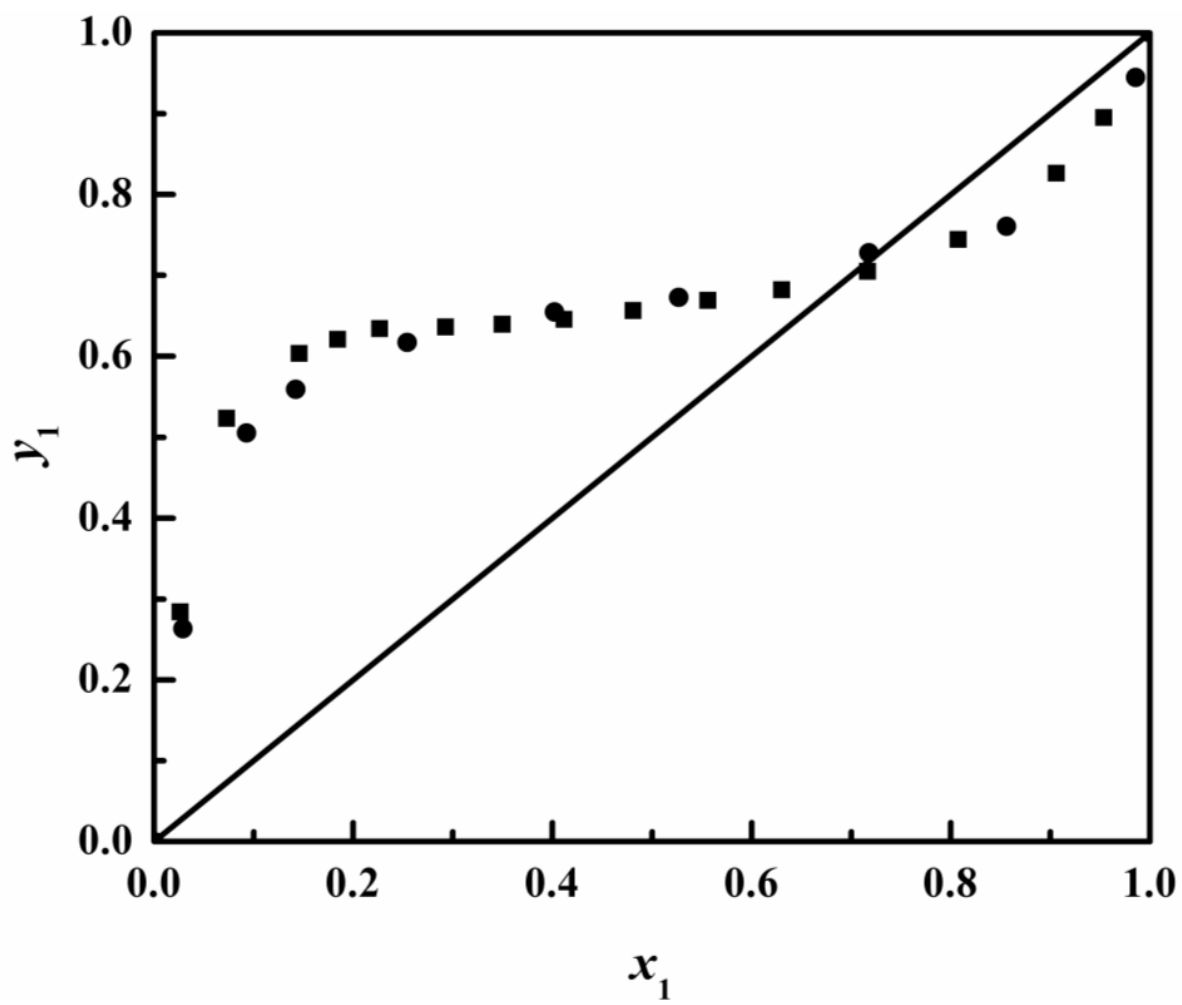

Figure S2. Comparison of experimental VLE for acetonitrile (1) + water (2) at $101.3 \mathrm{kPa}$ between this work (ø) and literature ${ }^{2}$ data (•) 


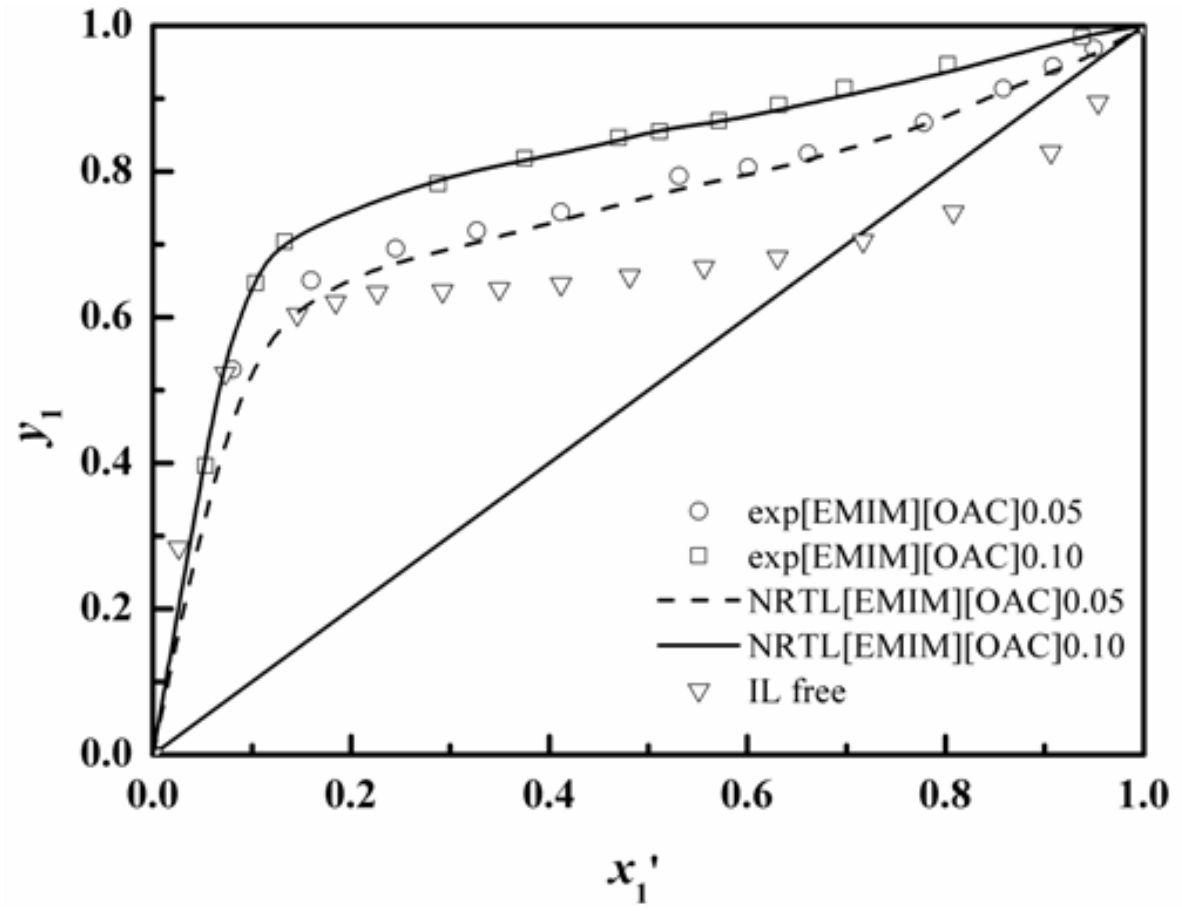

Figure S3. Isobaric VLE diagram for the acetonitrile (1) + water (2) + [EMIM][OAC] (3) systems at $101.3 \mathrm{kPa}$. 


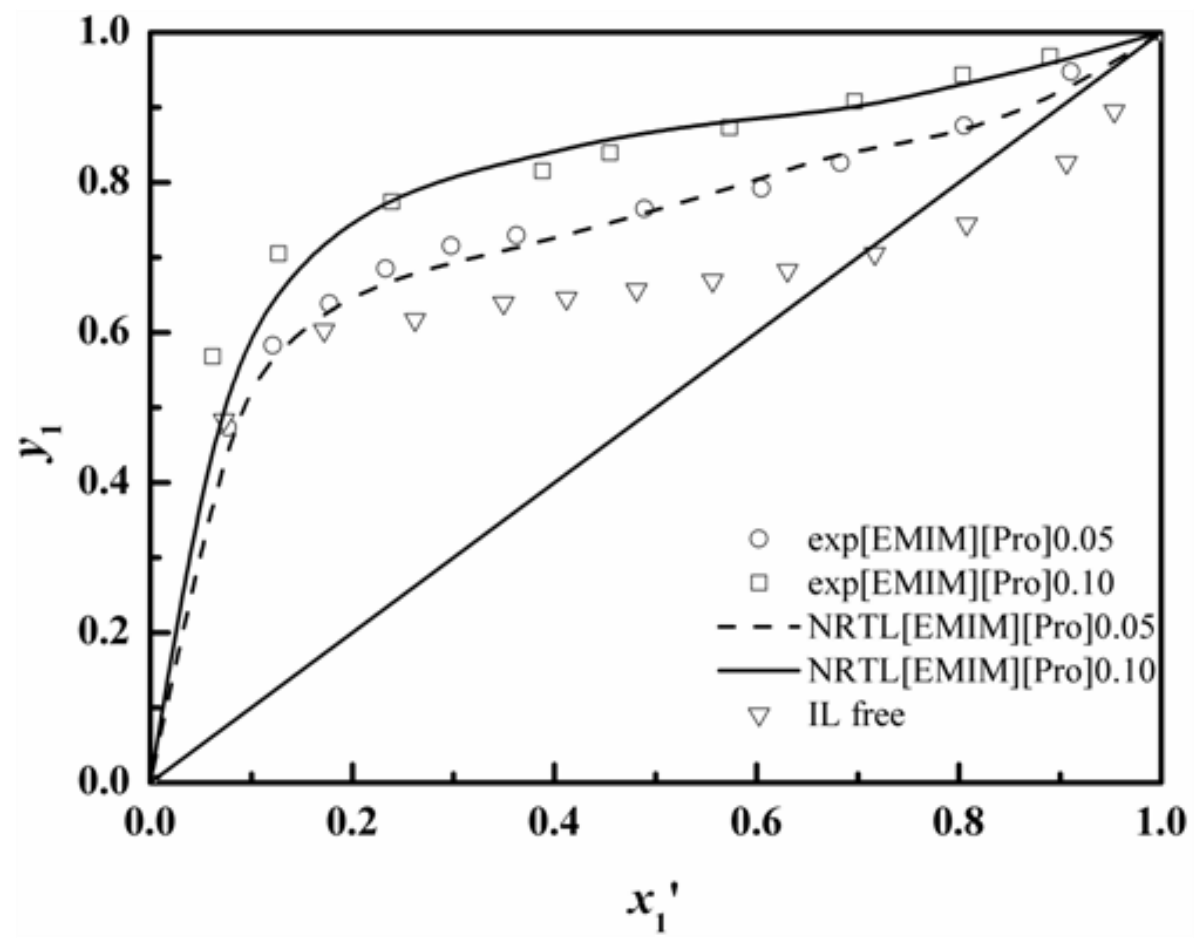

Figure S4. Isobaric VLE diagram for the acetonitrile (1) + water (2) + [EMIM][Pro] (3) systems at $101.3 \mathrm{kPa}$. 


\section{References}

(1) Bondi, A. van der Waals Volumes and Radii. J. Phys. Chem. 1964, 68, 441-451.

(2) Yin, W. C.;Cui, X. B.; Wu, T.; Zhang, X. K. Effect of ionic liquid on the vapor-liquid equilibrium of acetonitrile-water. Chem. Ind. Eng. Proc. 2009, 28, 190-193. (In Chinese)

(3) Tian, P. Green Solvent- Phase Equilibria and Micro-Structure of Ionic Liquids; China Science Precess: Beijing, 2009.

(4) Zhang, L. Z.; Qiao, B. B.; Ge, Y.; Deng, D. S.; Ji, J. B. Effect of ionic liquids on (vapor + liquid) equilibrium behavior of (water +2 -methyl-2-propanol). J. Chem. Thermodyn. 2009, 41, 138-143. 